CHINESE PHYSICS, Vol. 9, Number 2, pp. 124-130, February, 2000

\title{
Inversionless gain in a three-level system driven by a strong field and collisions *
}

\author{
A.K.Popov and S.A.Myslivets \\ Institute for Physics, Russian Academy of Sciences and Krasnoyarsk State University, \\ 660036 Krasnoyarsk, Russia. E-mail: popov@ksc.krasn.ru, fax: (3912)438923, \\ Gao Jin-yue and Zhang Han-zhuang \\ Department of Physics, Jilin University, Changchun 130023, China \\ B.Wellegehausen \\ Institut für Quantenoptik, Universität Hannover, Welfengarten 1, 30167 Hannover, Germany, \\ Fax:+49-511/762-2211,E-mail:Wellegehausen@mbox.iqo.uni-hannover.de
}

Received 14 March 1999

\begin{abstract}
Inversionless gain in a three-level system driven by a strong external field and by collisions with a buffer gas is investigated. The mechanism of populating of the upper laser level contributed by the collision transfer as well as by relaxation caused by a buffer gas is discussed in detail. Explicit formulae for analysis of optimal conditions are derived. The mechanism developed here for the incoherent pump could be generalized to other systems.
\end{abstract}

PACS numbers: $4250 ; 3150$

\section{INTRODUCTION}

Light amplification without population inversion (AWI) has been studied in a number of theoretical and experimental papers [1] - 32] because of its potential application in producing high-power lasers in the regions of electromagnetic spectrum which are difficult to reach with traditional laser system. Although population inversion between the laser levels is not necessary in inversionless light amplification system, it is required to pump a fraction of population to the upper level incoherently. Possible AWI at the transitions between excited states of noble gases, pumped by discharge, was shown in [1] 20]. The incoherent pump by discharge may not always provide necessary population, and the direct excitation by laser with broad linewidth may introduce additional atomic coherence. Another approach to possible AWI assisted by the collisional population transfer between fine structure levels of alkali atoms was proposed in [12, 13, 14] and based on successful experiments [33, 34] proved that wide range manipulation by population of the levels (up to population inversion) can be provided with this mechanism. Successful experiments on AWI in potassium vapors based on such mechanism were reported in [21]. (The theory and estimates for sodium atoms, underlying [12, 13, 14] were presented in [25, 28] too). Hyperfine structure of D-lines in alkalies and degeneracy of the coupled levels require multilevel model in order to consider complex of quantum nonlinear coherence and interference effects, which are origin of AWI. However experiments with sodium [22] and potassium 21] atoms showed, that major behavior and requirements for AWI (especially at detuning of the driving field from the resonance) may be studied with the aid of simple three-level V-configuration. In this paper, the mechanism of incoherent pump by a strong laser beam and collisional population transfer induced by buffer gas pressure is further analyzed. In order to fit better experimental conditions degeneracy are taken into account. We derived simple and explicit formulae, describing process. With the aid of these expressions we compare various alkalies and buffer gases in order to show that strong competition between population transfer and loss of coherence may be optimize by the proper choice of the transitions and buffer gases. The idea developed here for incoherent pump could be generalized to other systems.

\footnotetext{
* Project supported in part by the Russian Fundation for Basic Research (Grant No.96-02-00010c), by the National Sciences Foundation of China (Grant No. 19911121500) and the Science and Technology Foundation of Jilin Province, China (Grant No. 980526).
} 


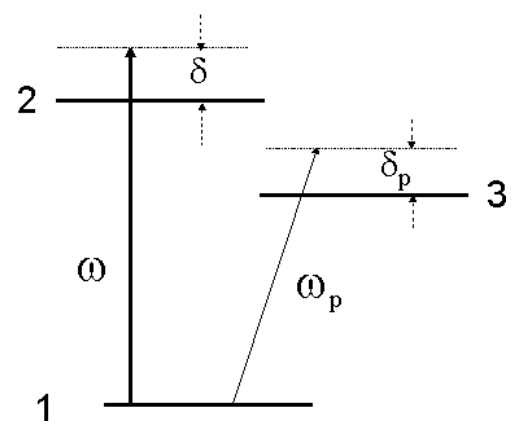

FIG. 1: Level scheme for inversionless amplification in the collision- driven three-level system. Transition $|3\rangle-|1\rangle$ is probed by the week radiation at the frequency $\omega_{p}$. Transition $|2\rangle-|1\rangle$ is coupled to the strong radiation at the frequency $\omega$. Level $|1\rangle$ is ground state, $|2\rangle$ and $|3\rangle$ are fine splitting levels. In order to manipulate population difference at the probe transition in the wide range from positive (absorption) via zero to negative values (gain), while maintaining absorption at the driven transition, we consider collisions with atoms of a buffer gas along with the saturation effects. Near Boltzmann's population distribution between fine-structure levels may set up due to the collisions.

\section{THEORETICAL MODEL AND EQUATIONS}

We consider a model as shown in Fig.1 which consists of three levels labeled $|1\rangle,|2\rangle$ and $|3\rangle$. A strong driving field with frequency $\omega$ produces coherent coupling between levels $|1\rangle$ and $|2\rangle$, while the weak probe field with frequency $\omega_{p}$ is scanned around the transition between levels $|3\rangle$ and $|1\rangle$. We express the probe and driving fields in the form:

$$
\begin{aligned}
E_{p}(t) & =\frac{1}{2}\left[E_{p 0} e^{-i \omega_{p} t}+c . c .\right] \\
E(t) & =\frac{1}{2}\left[E_{0} e^{-i \omega t}+c . c .\right]
\end{aligned}
$$

where $E_{p 0}$ and $E_{0}$ are the complex amplitudes. The Hamiltonian of the system including the interaction between the atom and the two fields can be written as:

$$
\begin{aligned}
H & =H_{a}+H_{b} \\
H_{a} & =\hbar \omega_{p} a_{3}^{+} a_{3}+\hbar \omega a_{2}^{+} a_{2} \\
H_{b} & =-\hbar \delta_{p} a_{3}^{+} a_{3}-\hbar \delta a_{2}^{+} a_{2}-\hbar\left[g_{p} a_{3}^{+} a_{1} e^{-i \omega_{p} t}+g a_{2}^{+} a_{1} e^{-i \omega t}+\text { c.c. }\right]
\end{aligned}
$$

where $\delta_{p}=\omega_{p}-\omega_{31}, \delta=\omega-\omega_{21} ; \omega_{31}$ and $\omega_{21}$ denote the transition frequencies from $|3\rangle$ to $|1\rangle$ and $|2\rangle$ to $|1\rangle$, respectively; $g=\mu_{21} E_{0} / 2 \hbar, g_{p}=\mu_{31} E_{p 0} / 2 \hbar$ are the Rabi frequencies of the probe and the driving fields, respectively; $\mu_{31}$ and $\mu_{21}$ are the dipole matrix elements of optical transitions from $|3\rangle$ to $|1\rangle$ and $|2\rangle$ to $|1\rangle$, respectively. In the interaction picture, the master equation for density operator is

$$
\frac{d r}{d t}=-\frac{i}{\hbar}\left[\tilde{H}_{b}, r\right]+\text { incoherent term }
$$

where $r=e^{i H_{a} t / \hbar} \rho e^{-i H_{a} t / \hbar}, \tilde{H}_{b}=e^{i H_{a} t / \hbar} H_{b} e^{-i H_{a} t / \hbar}$. According to the equation (四), the matrix elements of density operator can be expressed as follows:

$$
\begin{aligned}
& \frac{d r_{31}}{d t}=i g_{p}\left(r_{1}-r_{3}\right)-\left(\Gamma_{31}-i \delta_{p}\right) r_{31}-i g r_{32} \\
& \frac{d r_{32}}{d t}=i g r_{21}^{*}-i g^{*} r_{31}-\left[\Gamma_{23}-i\left(\delta_{p}-\delta\right)\right] r_{32} \\
& \frac{d r_{21}}{d t}=i g\left(r_{1}-r_{2}\right)-\left(\Gamma_{21}-i \delta\right) r_{21}-i g_{p} r_{32}^{*} \\
& \frac{d r_{3}}{d t}=2 \operatorname{Im}\left(g_{p}^{*} r_{31}\right)-\Gamma_{3} r_{3}+w_{23} r_{2} \\
& \frac{d r_{2}}{d t}=2 \operatorname{Im}\left(g^{*} r_{21}\right)-\Gamma_{2} r_{2}+w_{32} r_{3} \\
& r_{1}+r_{2}+r_{3}=1 .
\end{aligned}
$$


Here $r_{i j}=r_{j i}^{*}, r_{i i}=r_{i}, \Gamma_{2}=A_{21}+w_{23}, \Gamma_{3}=A_{31}+w_{32}$, where $A_{21}$ and $A_{31}$ are the decay rates of populations in levels $|2\rangle$ and $|3\rangle$ to the ground state $|1\rangle$ due to the common spontaneous transition, respectively; $w_{23}$ and $w_{32}$ are the collision transfer relaxation rates of the populations in level $|2\rangle$ to level $|3\rangle$ and in level $|3\rangle$ to level $|2\rangle$ due to collision; $\Gamma_{21}, \Gamma_{31}, \Gamma_{23}$ - are the polarization decay rates between levels $|1\rangle$ and $|2\rangle$, levels $|3\rangle$ and $|1\rangle$, levels $|3\rangle$ and $|2\rangle$, respectively $\left(\Gamma_{i j}=\Gamma_{j i}\right)$.

Absorption and refractive indices at the frequency $\omega_{p}$ are determined by the complex susceptibility $\chi\left(\omega_{p}\right)$ which is proportional to the off-diagonal element $r_{31}: \chi\left(\omega_{p}\right)=\left(r_{31} \mu_{13}\right) /\left(E_{p} / 2\right)$. In the liner approximation of probe field, the steady state solution of equations (5) for $r_{31}$ is:

$$
r_{31}=i \frac{g_{p}\left(r_{1}-r_{3}\right)-g r_{32}}{\Gamma_{31}-i \delta_{p}}
$$

The coherence $r_{32}$ itself is dependent on $r_{31}$ and $r_{21}$. By substituting solution for $r_{32}$ to equation(7) one obtains:

$$
\frac{\chi}{\chi_{0}}=f\left(\delta_{p}\right)=i \Gamma_{31} \frac{\left[\Gamma_{23}-i\left(\delta_{p}-\delta\right)\right]\left(r_{1}-r_{3}\right)-i g r_{21}^{*}}{\left[\Gamma_{23}-i\left(\delta_{p}-\delta\right)\right]\left(\Gamma_{31}-i \delta_{p}\right)+|g|^{2}}
$$

where

$$
r_{21}=-i g\left(r_{1}-r_{2}\right) /\left(\Gamma_{21}-i \delta\right)
$$

Eventually from (8),(9) we obtain:

$$
\begin{gathered}
f\left(\delta_{p}\right)=i \Gamma_{31} \frac{\left[\Gamma_{23}-i\left(\delta_{p}-\delta\right)\right]\left(r_{1}-r_{3}\right)-|g|^{2}\left(r_{1}-r_{2}\right) /\left(\Gamma_{21}+i \delta\right)}{\left[\Gamma_{23}-i\left(\delta_{p}-\delta\right)\right]\left(\Gamma_{31}-i \delta_{p}\right)+|g|^{2}}, \\
\alpha_{p} / \alpha_{p 0}=\operatorname{Im} f\left(\delta_{p}\right),\left(n_{p}-1\right) /\left(n_{p \max }-1\right)=\operatorname{Re} f\left(\delta_{p}\right)
\end{gathered}
$$

\section{MECHANISM OF POWER AND COLLISION DRIVEN $A W I$}

Optimum conditions for $A W I$ in three-level $V$ configuration, assuming possible manipulating by populations of the coupled levels with incoherent pump were analyzed in [1]. Classification of the effects of a strong field on the spectral line shape at an adjacent transition for various $V, \Lambda$ and $H$ configurations of coupled transitions was given in [1, 2] (see also [3] (e), 35]): dependencies of $r_{i, j}$ and of the denominator in (8) on $g$ refer to saturation of populations and energy-level splitting effects, correspondingly; $r_{21}$ - represents nonlinear interference effects $(N I E F)$. As it was shown in [2], 33(e) NIEF bring about change of a line shape but not an integral intensity:

$$
\int d \delta_{p} f\left(\delta_{p},|E|^{2}\right)=\pi \Gamma_{31}\left(r_{1}-r_{3}\right),
$$

which depends only on saturation effects.

Indeed, NIEF represent the origin of sign-changing spectral line shapes and AWI at the probe transition. One can regard (8) as the difference between acts of pure emission (associated with the $r_{3}$, assuming $r_{1}=$ $r_{2}=0$ ), and pure absorption (the rest terms). Both of them are positive but depend in a different way on detunings, because of NIEF. This was emphasized in [2] (see also [3] (e), 1, 35).

Thus, in the schematic under consideration $A W I$ originates from the coherence at the transition $|3\rangle-|2\rangle$, induced by the strong field, coupled to the auxiliary level $|2\rangle$ in combination with the probe field, which consequently gives rise to factor $r_{21}$ in (8). The larger is maximum value of $r_{21}$ compared to $r_{1}-r_{3}$ the more pronounced is the effect of $A W I$. At $\delta=0$, maximum of absorption (gain) corresponds to $\delta_{p}=0$.

$$
f(0)=\frac{\left(r_{1}-r_{3}\right)-\left(r_{1}-r_{2}\right) S}{1+S}
$$

where $S=|g|^{2} / \Gamma_{21} \Gamma_{32}$. Therefore, even at $\left(r_{1}-r_{3}\right)>0,\left(r_{1}-r_{2}\right)>0$, negative absorption (gain) occurs if

$$
\left(r_{1}-r_{2}\right) S>r_{1}-r_{3} \text {. }
$$

The less is coherence decay rate $\Gamma_{32}$ at the two-photon transition $|3\rangle-|2\rangle$, compared to that at the coupled 
$|1\rangle$ on two quasi levels significantly reduces interference and, therefore, the magnitude of AWI at the center of the transition $|3\rangle-|1\rangle$. Optimal value of strength of driving field for $A W I$ in the probe line center was analyzed in 迫.

Below we shall consider opportunities and specific experimental schematic to control shape of absorption and refraction indexes without external incoherent pump. Necessary for $A W I$ distribution of populations is ensured by collisions.

Consider alkali atoms, immersed into buffer gas. Strong field couples $P_{3 / 2}$ and ground $S$ levels. Fast collision exchange ensures population transfer from the $P_{3 / 2}$ to the lower $P_{1 / 2}$ level. In order to understand main mechanism giving rise to $A W I$, suppose for simplicity, that pressure of the buffer gas is so strong, that Boltzmann population distribution between the fine structure levels is established. This brings about the means to control population difference at the probe transition in a wide range by increasing intensity of the strong field. Even population inversion at the $P_{1 / 2}-S$ transition can be provided due to the saturation effect at $P_{3 / 2}-S$ transition (similar to that in Ruby laser). Such a feasibility to produce population inversion at the transition $P_{1 / 2}-S$ has been demonstrated experimentally for potassium [33] and sodium [34 vapors admixed to helium at about atmospheric pressure. Possible $A W I$, the required driving field strength and buffer gas pressure can be estimated as follows. One can find from the equation system (6):

$$
\begin{aligned}
& r_{2}=\frac{\Gamma_{3}}{2 \Gamma_{3}+w_{23}} \cdot \frac{\mathfrak{x}^{\prime}}{1+\mathfrak{x}^{\prime}}, r_{3}=\frac{w_{23}}{\Gamma_{3}} r_{2}, r_{1}=\left(1+\frac{\Gamma_{3} æ^{\prime}}{2 \Gamma_{3}+w_{23}}\right) \frac{1}{1+\mathfrak{x}^{\prime}} ; \\
& r_{1}-r_{2}=\frac{1}{1+\mathfrak{x}^{\prime}}, r_{1}-r_{3}=\left(1+\frac{\Gamma_{3}-w_{23}}{2 \Gamma_{3}+w_{23}} \mathfrak{x}^{\prime}\right) \frac{1}{1+\mathfrak{x}^{\prime}} .
\end{aligned}
$$

Here

$$
\begin{aligned}
& æ^{\prime}=\frac{2+\left(w_{23} / \Gamma_{3}\right)}{\left[1-\left(w_{32} w_{23} / \Gamma_{3} \Gamma_{2}\right)\right.} \cdot æ, \\
& æ=2 \Gamma|g|^{2} / \Gamma_{2}\left(\Gamma^{2}+\delta^{2}\right)
\end{aligned}
$$

Consider high pressure limit, assuming that $A_{31} \simeq A_{21}$, and the buffer gas pressure is so high, that $\left(w_{23}-w_{32}\right)>>A_{31}, A_{21}$. Then taking into account that in the given conditions $w_{32}=w_{23} \exp \left(-\Delta E / k_{B} T\right)$, where $\Delta E=E_{2}-E_{3}$ is the fine splitting energy, $k_{B}$ and $T$ are Boltzmann's constant and temperature, we obtain:

$$
\begin{array}{r}
r_{1}-r_{3}=\left[1-\Re^{\prime} \frac{1-\exp \left(-\Delta E / k_{B} T\right)}{1+2 \exp \left(-\Delta E / k_{B} T\right)}\right] \frac{1}{1+\Re^{\prime}} ; \\
\Re^{\prime}=\frac{1+2 \exp \left(-\Delta E / k_{B} T\right)}{1+\exp \left(-\Delta E / k_{B} T\right)} \cdot \frac{2|g|^{2} \Gamma_{21} / A_{21}}{\Gamma_{21}^{2}+\delta^{2}} .
\end{array}
$$

For sodium $\Delta E=17.2 \mathrm{~cm}^{-1}$ and at $T=550 \mathrm{~K}$ the estimates give

$$
\begin{gathered}
\Delta E / k_{B} T=4.3 \cdot 10^{-2}, \quad x^{\prime} \simeq 3|g|^{2} / \Gamma \Gamma_{2} \simeq 9 \lambda^{3} I / 64 \pi^{3} \epsilon_{0} \hbar c \Gamma_{21}, \\
r_{1}-r_{3} \simeq \frac{1}{1+\mathfrak{x}^{\prime}}\left[1-1.3 \cdot 10^{-2} \mathfrak{x}^{\prime}\right]
\end{gathered}
$$

Here $\lambda$ and I are strong field wavelength and energy flux density, $\epsilon_{0}$ is primitivity of free space. From the equations $(12),(18),(19)$ ) one can see that the potentially attainable AWI grows with the increase of $\Delta E$ (for example, in $K$ and $R b$ ). But on the other hand with the increase of fine splitting population transferring collision crossection decreases. That must be compensated with increase of buffer gas pressure. Later brings loss of coherence. These dependencies will be investigated numerically in the next section.

Inelastic collision crossection of sodium and helium for the transition $3 P_{3 / 2}-3 P_{1 / 2}$ is $\sigma_{23} \simeq 4 \cdot 10^{-15}$ $\mathrm{cm}^{2}$ [36. For $\mathrm{T}=550 \mathrm{~K}$ and atmospheric pressure of helium we estimate $w_{23}=N_{H e} \bar{v} \sigma_{23} \simeq 7.5 \cdot 10^{9} \mathrm{sec}^{-1}$. Since $A_{31} \simeq A_{21} \simeq 6.2 \cdot 10^{7} \mathrm{sec}^{-1}$, requirements for the approximations (18), (19) are met. Taking the data of ref. 37] for the collision broadening of sodium $D$ lines by helium, we estimate collision halfwidth as $\Gamma_{21} \simeq 5 \cdot 10^{10} \mathrm{sec}^{-1}$, which exceeds measured Doppler halfwidth of this transition $\Delta \omega_{D} / 2=4.7 \cdot 10^{9} \mathrm{sec}^{-1}$ $\left(\Delta \nu_{D} / 2=0.75 \mathrm{GHz}\right)$. So we can neglect inhomogeneous broadening of the transition.

For the conditions under consideration we estimate $\mathfrak{x}^{\prime} \simeq 5 \cdot 10^{-9} \cdot I$ (where I is in W/ $\left.\mathrm{cm}^{2}\right),|g|^{2} / \Gamma_{21} \Gamma_{32} \simeq$ $|a|^{2} / \Gamma_{31} \Gamma_{32} \simeq x^{\prime} \Gamma_{2} / 3 \Gamma_{32}$. For the laser power $0.1 \mathrm{~W}$ focused to the spot $A=10^{-5} \mathrm{~cm}^{2}$ (confocal parameter 
$b \simeq 1 \mathrm{~cm})$ we obtain $|g| \simeq 3.6 \mathrm{GHz}, x^{\prime} \simeq 5 \cdot 10^{2},|g|^{2} / \Gamma_{21} \Gamma_{32} \simeq 0.1$. These magnitudes are about optimal ones and correspond to the estimated values, required to vary population difference at the probe transition $r_{1}-r_{3}$ around zero. Above presented estimates for the intensity $(1-10) \mathrm{kW} / \mathrm{cm}^{2}$, required to achieve appreciated change of the line shape under the conditions considered, compares well with the experimental data obtained for the significant change of the ratio of population differences at the coupled transitions at the similar conditions [34].

The magnitude of AWI at $r_{1}-r_{3}=0$ is estimated as $\alpha_{p}(0) / \alpha_{p 0} \simeq \Gamma_{2} / 3 \Gamma_{32}$. Assuming $\Gamma_{32} \simeq w_{23}$, it may yield about $0.3 \%$ of the absorption in the absence of the strong field. It is seen that this quantity is very sensitive to the decay rate of the coherence at the Raman-like transition $|3\rangle-|2\rangle$.

\section{NUMERICAL ANALYSIS OF POWER AND COLLISION INDUCED AWI AT THE TRANSITION BETWEEN DEGENERATE LEVELS OF ALKALIES}

We shall consider such frequency detunings and collision broadening of the transitions, that hyperfine splitting of the levels can be neglected. However, account for degenerating of the levels may occur important for the quantitative analysis of the optimum conditions for $A W I$.

Assume that both driving and probe fields are linear and in the same way polarized. Then we assume that collisions can transfer only populations but not coherence. By that we can consider equations (5) for off-diagonal elements of density matrix as referred to the transitions between the same Zeeman sublevels of the upper and lower energy levels, which are not coupled. Unlike that equations for populations of the sublevels are coupled by the collisions. We assume that collisions are so strong, that populations of all sublevels within a same level are equal. Consequently we shall consider populations of any sublevel $r_{i M}$ and of the level $R_{i}$ to be related as $r_{i M}=R_{i} / g_{i}$, where $g_{i}$ is degenerating factor of the level. Equations for the populations take the form:

$$
R_{2}=\left(\frac{g_{2}}{g_{1}} R_{1}-R_{2}\right) æ+R_{3} \frac{w_{32}}{\Gamma_{2}} ; R_{3}=R_{2} \frac{w_{23}}{\Gamma_{3}} ; R_{1}+R_{2}+R_{3}=1,
$$

where $æ$ is given by (17). Solution of (21) is:

$$
\begin{aligned}
& R_{2}=\frac{g_{2} / g_{1}}{1+\left(g_{2} / g_{1}\right)\left[1+\left(w_{23} / \Gamma_{3}\right)\right]} \cdot \frac{\mathfrak{x}^{\prime}}{1+\mathfrak{x}^{\prime}} ; R_{3}=R_{2} \frac{w_{23}}{\Gamma_{3}} ; \\
& R_{1}=\left[1+\frac{\mathfrak{x}^{\prime}}{1+\left(g_{2} / g_{1}\right)\left[1+\left(w_{23} / \Gamma_{3}\right)\right]}\right] \cdot \frac{1}{1+\mathfrak{x}^{\prime}}, \\
& \frac{R_{1}}{g_{1}}-\frac{R_{2}}{g_{2}}=\frac{1}{g_{1}} \cdot \frac{1}{1+\mathfrak{x}^{\prime}}, \\
& \frac{R_{1}}{g_{1}}-\frac{R_{3}}{g_{3}}=\frac{1}{g_{1}}\left\{1+\frac{\left[1-\left(g_{2} w_{23} / g_{3} \Gamma_{3}\right)\right] \mathfrak{x}^{\prime}}{1+\left(g_{2} / g_{1}\right)\left[1+\left(w_{23} / \Gamma_{3}\right)\right]}\right\} \cdot \frac{1}{1+\mathfrak{x}^{\prime}} \text {. }
\end{aligned}
$$

It is seen from (24) that population inversion at the probe transition is impossible until buffer gas number density $N_{b}$ meets requirement:

$$
N_{b}\langle v\rangle\left(g_{2} \sigma_{23}-g_{3} \sigma_{32}\right)<g_{3} A_{31}
$$

\begin{tabular}{|c|c|c|c|c|}
\hline Atom & $\sigma_{P_{3 / 2}-P_{1 / 2}}\left(\AA^{2}\right)$ & $\sigma_{P_{1 / 2}-P_{3 / 2}}\left(\AA^{2}\right)$ & $\sigma_{S_{1 / 2}-P_{3 / 2}}\left(\AA^{2}\right)$ & $\sigma_{S_{1 / 2}-P_{1 / 2}}\left(\AA^{2}\right)$ \\
\hline $\mathrm{Na}$ & 41.140 & 7740 & 15939 & $137[39]$ \\
\hline K & 52.840 & 8440 & 13339 & 100 39. \\
\hline $\mathrm{Rb}$ & $0.12[40]$ & 0.140 & $145[39$ & $145[39$ \\
\hline
\end{tabular}

Here $\langle v\rangle$ and $\sigma_{i j}$ are averaged relative collision velocity and fine structure population transfer crossections with the buffer atom.

For numerical analysis we use data presented in Table 1 (with $\mathrm{He}$ as buffer gas). Fig.2 (curves a)

TABLE I: Collision cross-sections

shows dependence of threshold value of the saturation parameter $æ_{0}$, required for population inversion to be achieved, on the buffer gas pressure above this limit (equation $\left.\left(R_{1} / g_{1}\right)-\left(R_{3} / g_{3}\right)=0\right)$. Curves b show dependence of threshold value of the saturation parameter $æ_{0}$, required in order to achieve inversion of sign of $\alpha_{p}(A W I)$, on the buffer gas pressure (equation $\left.\left[\left(R_{1} / g_{1}\right)-\left(R_{3} / g_{3}\right)\right]-\left[\left(R_{1} / g_{1}\right)-\left(R_{2} / g_{2}\right)\right] S=0\right)$. Both dependencies are drown for atoms at $v=0$ and at $\delta=\delta_{n}=0$ æ $x_{0}$ is resonant value for saturation parameter 

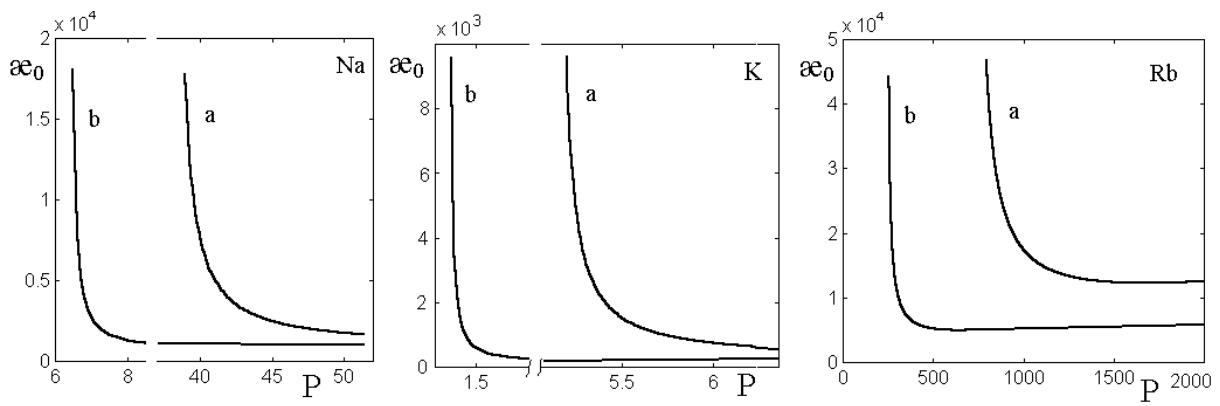

FIG. 2: Threshold value of the saturation parameter $æ_{0}$, required for population inversion to be achieved, vs buffer gas $(\mathrm{He})$ pressure (a). Threshold value of the saturation parameter $æ_{0}$, required for inversion of sign of $\alpha_{p}(A W I)$ (AWI) to be achieved, vs $H e$ pressure (b). Both dependencies are drown for atoms at $v=0$ and at $\delta=\delta_{p}=0$. æ⿸ is resonant value for saturation parameter at collisionless limit $\left(æ_{0}=4|g|^{2} / A_{21}^{2}\right)$.

at collisionless limit $\left(æ_{0}=4|g|^{2} / A_{21}^{2}\right)$. In the range between curves (a) and (b) change of sign of $\alpha_{p}$ occurs without changing of sign of population difference $\left(R_{1} / g_{1}\right)-\left(R_{3} / g_{3}\right)$. Minimum value of the saturation parameter $æ_{0}$, required in order to achieve AWI is $æ_{0}=92\left(P_{b} \simeq 3.1\right.$ Torr $), æ_{0}=848\left(P_{b} \simeq 12.4\right.$ Torr $)$ and $æ_{0}=4971$ ( $P_{b} \simeq 640.5$ Torr $)$ for $K, N a$ and $R b$ respectively. Such values of the saturation parameter lead to considerable change of level populations which becomes $R_{1}=0.28, R_{2}=0.5, R_{3}=0.22$ for $K, R_{1}=0.264$, $R_{2}=0.51, R_{3}=0.226$ for $N a$ and $R_{1}=0.324, R_{2}=0.462, R_{3}=0.214$ for $R b$.

One can manipulate the shape of the absorption/gain spectral line, position and bandwidth of the absorption and gain frequency-intervals. Analysis is given in ref. [1]. Line shape is quite sensitive to the intensity and frequency detunings of the strong field from the resonance. Bandwidth of the gain grows and the maximum value decreases with the increase of the intensity of the strong field above a certain magnitude. Saturation of the population difference at the strong field transition and splitting of the common energy level oppose AWI effect, so that it can be optimize by the proper choice of intensity and detunings of the strong field. In the case under consideration inelastic collision frequency is important parameter to be optimized as well.

Fig.3 demonstrate dependencies of absorption/gain of probe field at frequency $\omega_{p}$ vs scaled detuning $\delta_{p} / \Gamma_{31}$ $(\delta=0)$ under optimal values of the buffer gas pressure and of the driving field intensity. Maximum values of the AWI index are: $\alpha_{p}=0.002$ for $N a\left(æ_{0}=8700, P=170\right.$ Torr $) ; \alpha_{p}=0.01$ for $K\left(æ_{0}=370, P=16\right.$ Torr) and $\alpha_{p}=1.3 \cdot 10^{-4}$ for $R b\left(æ_{0}=12300, P=1800\right.$ Torr $)$. Corresponding population differences $\left(R_{1} / g_{1}\right)-\left(R_{3} / g_{3}\right)$ are $2 \cdot 10^{-5}, 3 \cdot 10^{-4}$ and $1.2 \cdot 10^{-4}$.

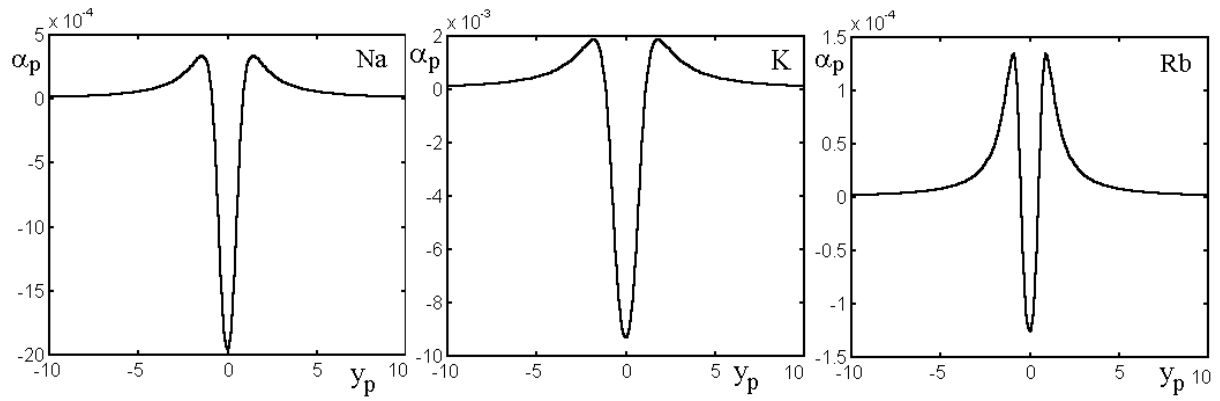

FIG. 3: Line shape of absorption(positive)/gain(negative) index $\alpha_{p}\left(\delta_{p}\right)$ for the probe field at the $D_{1}$ transition in the presence of a strong laser field, resonant to the $D_{2}$ transition. Abscisse: detuning of the probe laser in units of collision-broadened halfwidth $\delta_{p} / \Gamma_{31}$. Ordinate: index scaled to the absorption maximum in absence of the driving field. Strong field is tuned to exact resonance. Curves correspond to near zero population difference at the probe transition, so that line shapes are almost completely determined by the NIEF. Intensity of the strong field is such that Rabi frequency $g=2.9 \cdot 10^{9} \mathrm{sec}^{-1}(N a), g=0.37 \cdot 10^{9} \mathrm{sec}^{-1}(K)$ and $g=2.2 \cdot 10^{9} \mathrm{sec}^{-1}$ (Rb) so that population difference at the probe transition $|1\rangle-|3\rangle$ is still positive.

Taking the data of ref. [39] for the collision broadening of $D$ lines by helium, we estimate collision halfwidth as $\Gamma_{21} \simeq 8.8 \cdot 10^{9} \mathrm{sec}^{-1}, \Gamma_{21} \simeq 0.73 \cdot 10^{9} \mathrm{sec}^{-1}$ and $\Gamma_{21} \simeq 79.8 \cdot 10^{9} \mathrm{sec}^{-1}$ for $N a, K$ and $R b$ respectively, which exceeds measured Doppler halfwidth of this transition for $N a\left(\Delta \omega_{D} / 2=5.6 \cdot 10^{9} \mathrm{sec}^{-1}\right)$ and $R b$ $\left(\Delta \omega_{D} / 2=2.15 \cdot 10^{9} \mathrm{sec}^{-1}\right)$. So in this case we can neglect inhomogeneous broadening of the transition. But in the case of $K \Gamma_{21} \ll \Delta \omega_{D} / 2=3.1 \cdot 10^{9}$ and it is necessary to perform averaging over velocities of atoms.

Fig. 4 shows velocity-averaged absorption indices (for $K$ ) at inhomogeneously broadened $|1\rangle-|3\rangle$ transition 


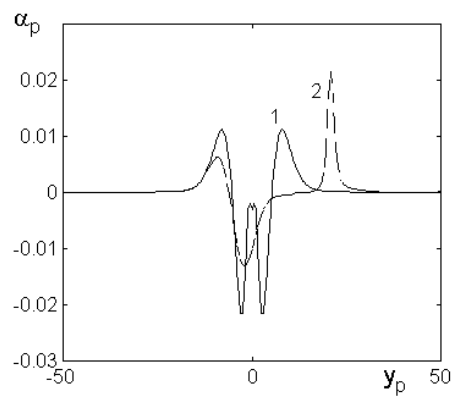

FIG. 4: Velocity-averaged absorption index (for $K$ ) at inhomogeneously broadened $|1\rangle-|3\rangle$ transition in the presence of driving field at $|1\rangle-|2\rangle$ transition $\left(1-æ_{0}=3400, \delta=0,2-æ_{0}=6 \cdot 10^{4}, \delta=3 \cdot \Delta \omega_{D} / 2\right)$.

in the presence of strong field at $|1\rangle-|2\rangle$ transition $\left(1-\mathfrak{x}_{0}=3400, \delta=0,2-\mathfrak{x}_{0}=6 \cdot 10^{4}, \delta=3 \cdot \Delta \omega_{D} / 2\right)$.

The shape of the refraction index (dispersion) is described by the $\operatorname{Re} f\left(\delta_{p},|E|^{2}\right)$. In the scope of the discussed experiment one can manipulate by the shape of both absorption (gain) and refraction indexes so, that maxima and minima of the refraction would fall to the spectrum range of the near vanishing absorption.

In summary, this work considers a model of interference and collision driven $V$ - type three degeneratelevel system which, provides gain without population inversion and resonance-enhanced refraction at vanishing absorption. Explicit formulas for analyzing of the optimal conditions are presented. Comparative analysis of use of various alkalies relative to potential experiment is qualitatively discussed in details. Coherence destroying collision decrease the AWI and ERWA effects compared to that in atomic beams. However the decrease may be comparable to that due to the Doppler broadening in metal vapors. The advantages are simplicity of the experiment, feasibilities to manipulate the population differences at the coupled transitions and to avoid some side effects. These make the experiment conformable to the simple common accepted theoretical model.

[1] T.Ya.Popova, A.K.Popov, Zh.Prikl.Spektr. (USSR) 12, 989(1970) [Translated in Engl.: Journ. Appl. Spectr 12, No 6, 734, (1970), quant-ph/0005047; Izv.VUZ. Fiz. (USSR) No 11, 38 (1970) Izv.Vysh. Uchebn. Zaved., Fizika No 11, 38, (1970) [Translated in Engl.: Soviet Phys. Journ. 13, No 11, 1435, (1970)], quant-ph/0005049; A.K.Popov. Vvedeniye v Nelineinuyu Spektroskopiyu (Introduction to Nonlinear Spectroscopy) (in Russ.), Novosibirsk, Nauka, 1983.

[2] T.Ya.Popova, A.K.Popov, S.G.Rautian, R.I. Sokolovsky, ZhETF 57, 850 (1969) (Sov.Phys.JETP. 30466 (1970)) quant-ph/0005094

[3] For the survey of early publications of Russian groups on AWI see for example $a$. S.G.Rautian and I.I.Sobelman, Zh. Eksp. Teor. Fiz., 41, 456, 1961; b.D.N.Klyshko, Yu.S.Konstantinov, V.S.Tumanov, Izv. Vuz. Radiofiz., 8, 513, 1965; c.A.M.Bonch-Bruevich, S.G.Przhibelskii, N.A.Chigir, Vestnik MGU, Fisika, 33, N4, 35 (1978) (Engl.:p2835); d.I.S.Zelikovich, S.A.Pulkin, L.S.Gaida, V.N.Komar, Zh. Eksp. Teor. Fiz., 94, 76, 1988; e. S.G.Rautian and A.M.Shalagin, Kinetic Problems of Nonlinear Spectroscopy (North Holland, 1991).

[4] O. Kocharovskaya, Y. L. Khanin, JETP Lett. 48, 630 (1988)

[5] M. O. Scully, S. Y. Zhu, A. Gavrielides, Phys. Rev. Lett. 62, 2813 (1989)

[6] S. E. Harris, Phys. Rev. Lett. , 62, 1022 (1989)

[7] L. M. Narducci, H. M. Doss, P. Ru, M. O. Scully, S. Y. Zhu, C. Keitel, Optics Comm. 81,379 (1991)

[8] O. Kocharovskaya, Phys. Rep. 219, 175 (1992)

[9] M. O. Scully, Phys. Rep. 219, 191 (1992)

[10] M. Fleischhauer, C. H. Keitel, M. O. Scully, Phys. Rev. A 46, 1468 (1992)

[11] J. Y. Gao, C. Guo, X. Z. Guo, G. X. Jin, Q. W. Wang, J. Zhao, H. Z. Zhang, J. Jiang, D. Z .Wang, D. M. Jiang, Opt. Commun. 93, 323 (1992)

[12] Popov A.K. and Wellegehausen B. Interference and cw Gain without Population Inversion in Three-Level Systems.: In Scientific Program of Euroconference on LICS and Related Topics, Crete, Greece, May, 1993.

[13] Müller-Wirts Th., Eichmann H., Popov A.K. and Wellegehausen B. Gain without Population Inversion at Homogeneously Broadened $D_{1}$ Line of Sodium.- In Proc.EQEC'93, Florence, Italy, Sept. 1993, PP. 23, 318-320.

[14] Müller-Wirts Th., Wellegehausen B. and Popov A.K. Experiments on $N a$ Vapors, Related to Lasing with(out) Inversion.- In Scientific Program of Crested Butte Workshop on Effects of Atomic Coherence and Interference. The Woodlands, Texas, U.S.A., 1993.

[15] U. Rathe, M. Fleischhauer, S. Y. Zhu, Phys. Rev. A 47,4994 (1993)

[16] A. Nottelman, C. Peters, W. Lange, Phys. Rev. Lett. 70, 1783 (1993) 
[17] W. E. Van der Veer, R. J. J. Van Diest, A. Donszelmann, H. B. Van Linden van den Heuvell, Phys. Rev. Lett. 70, $3243(1993)$

[18] E. Fry, X. Li, D. Nikonov, G. G. Padmabandu, M. O. Scully, A. V. Smith, F. K. Tittel, C. Wang, S. R. Wilkinson, S. Y. Zhu, Phys. Rev. Lett. 70, 3225 (1993)

[19] A.K. Popov, V.G. Arkhipkin, Th. Müller-Wirts and B. Wellegehausen, Interference processes and gain without inversion in three level schemes, Paper QThA2. In Proc. CLEO/IQEC'94, Anaheim, CA, USA, May, 1994 , p.105.

[20] Th. Müller-Wirts, M. Dernier, A.K. Popov and B. Wellegehausen, Paper QMG7-In Proceed. CLEO/Europe EQEC, Amsterdam, 1994.

[21] J. A. Kleinfeld, A. D. Streater, Phys. Rev. A 49, R4301 (1994)

[22] J. Y. Gao, H. Z. Zhang, H. F. Cui, X. Z. Guo, Y. Jiang, Q. W. Wang, G. X. Jin, J. S. Li, Optics Commun. 110, $590(1994)$

[23] M. Xiao, Y. Q. Li, J. Gea-Banacloche, Phys. Rev. lett. 74, 666 (1995)

[24] A.S.Zibrov, M.D.Lukin, D.E.Nikonov, L.Hollberg, M.O.Scully, V.L.Velichansky, and H.G.Robinson, Phy.Rev.lett. 75, 1499 (1995)

[25] A.K.Popov, Izvestija of Akademii Nauk (in Russ). 60. No6. pp 99-120, 1996 [Engl.: Bull. Russ Acad. Sci. (Ser. Phys.), 60, No6, pp 927-945, 1996] (review), quant-ph/0005108

[26] A.K.Popov and S.G.Rautian, in Coherent Phenomena and Amplification without Inversion. A. V. Andreev, O. Kocharovskaya and P. Mandel, eds., Proc. SPIE v.2798, 49-61 (1996) (invited paper), quant-ph/0005114

[27] A.K.Popov, V.M.Kuchin and S.A.Myslivets, Zh.Eksp. Teor. Fiz., 113, No2, 445-470 (1998) [Engl. JETP, 86, No2, 244-258 (1998)].

[28] A.K.Popov and B.Wellegehausen Laser Physics (Moscow), 6, No 2, 364-371, (1996);

[29] Hong Yuan Ling, Yong-Qing Li, and Min Xiao, Phys. Rev. A 53, 1014 (1996)

[30] Han-Zhuang Zhang, Jin-Yue Gao, Z.Phys.D, 38, 5 (1996)

[31] A. S. Zibrov, M. D. Lukin, L. Hollberg, D. E. Nikonov, M. O. Scully, H. G. Robinson, and V. L. Velichansky, Phys. Rev. Lett. 76, 3935 (1996)

[32] O. Schmidt, R. Wynands, Z. Hussein, and D. Meschede, Phys. Rev. A, 53, R27 (1996)

[33] B. A. Glushko, M.E. Movsesyan, T.O. Ovakimyan, Opt. i Spektrosk. (USSR) 54, 762 (1982) [Engl.: Opt. Spectrosc. 54, N4 (1982)].

[34] S.N.Atutov, A.I.Plekhanov, A.M.Shalagin, Opt. i Spektrosk. (USSR) 56, 215 (1984) (Opt. Spectrosc. 56, 134 (1984).)

[35] Yu.I.Heller and A.K.Popov, Laser Induction of Nonlinear Resonances in Continuous Spectra. Novosibirsk, Nauka, 1981 (in Russ.) [Engl.transl.: Journ. Sov. Laser Research, 6, No 1-2, Jan.-Feb., 1985, Plenum, c/b Consultants Bureau, NY, USA].

[36] J.A. Jordan and P.A.Franken, Phys. Rev., 142, 20 (1966)

[37] J.F.Kielkopf, J.Phys., B13, 3813 (1980)

[38] N. Allard and J. Kielkopf, Rev. Mod. Phys. 54, 1103 (1982)

[39] E. L. Lewis, Phys. Rep. 58, 1(1980)

[40] Beahn T.J., Condell W.J., Mandelberg H.I. Phys. Rev., 141, 83 (1966) 